\title{
On Convexity and Approximating the Perimeter of an Ellipse
}

\author{
Octav Olteanu \\ Department of Mathematics-Informatics, University Politehnica of Bucharest, Bucharest, Romania \\ Email: olteanuoctav@yahoo.ie
}

Received 20 February 2014; revised 24 March 2014; accepted 7 April 2014

Copyright (C) 2014 by author and OALib.

This work is licensed under the Creative Commons Attribution International License (CC BY). http://creativecommons.org/licenses/by/4.0/

c) (i) Open Access

\begin{abstract}
In the first part of this work, a convex partition of a compact subset is constructed. Minimumlength surrounding curve and minimum-area surrounding surfaces for a compact set are constructed too. In the second part, one writes the perimeter of an ellipse as the sum of an alternate series. On the other hand, we deduce related "sandwich" inequalities for the perimeter, involving Jensen's inequality and logarithmic function respectively. We discuss the values of the ordinate of the gravity center of the upper semiellipse at the ends of the positive semiaxes, in terms of the scale ratio $b / a$.
\end{abstract}

\section{Keywords}

Convexity, Minimum Length, Approximation, Inequalities, Perimeter of the Ellipse

\section{Introduction}

Classical and relative recent evaluations and inequalities for the perimeter of the ellipse are recalled and proved in [1]-[3]. We continue this type of results by writing the perimeter of the ellipse as a sum of an alternate series. One proves two sandwich-type inequalities. The first one involves irrational functions of the semiaxes, while the second one uses the logarithmic function. Many authors have studied the applications of results on non-convex compact spaces. This paper shows how to decompose an arbitrary compact set into its "convex components". Based on the convexity of the subsets, we can then derive properties of the whole non-convex set. Strassen has presented a disintegration theorem for representing a function dominated by a sum, as a sum of functions with some properties [4]. The current paper shifts the discussion from functions to sets and presents the decomposition of a set into convex sets. The general idea is similar to the partition of an open subset, formed by its connected components.

Next, I consider the convex hull of a compact set and propose a minimal-length surrounding curve and mini- 
mum-area surrounding surface for a compact set. In [5], a similar minimal surface problem is considered in the setting of differential geometry and calculus of variations. Local affine approximation methods are used in [6] [7]. For practical reasons, in this work I use smooth approximations of convex (or concave) maps obtained from the convex hull (see also [8]). The importance of Krein Milman and Caratheodory's theorems is pointed out in [6] [7] [9], and in the present work too. Jensen's inequality [9] plays a central role in analysis, with applications in other fields. It appears in this work, related to the perimeter of the ellipse. The following works deal with smooth approximation, not only on compact, but also on unbounded subsets of $R^{n}$ : [8] [10].

The background for this work is contained in [6] [9] [11]. The present work follows the way of real analysis, as well as elements of topology and complex analysis. The paper is organized as follows. In Section 2, we point out convex partitions of a compact subset. Section 3 deals with the minimal-length surrounding curve and minimal area surrounding surface. In Section 4, I introduce three types of approximations for the perimeter of the ellipse. Section 5 concludes the paper.

\section{Constructing Convex Partition of a Compact Set}

The following theorem shows the decomposition of a compact non-convex set into convex subsets. The proof not only shows the existence of the decomposition but also gives a method for constructing the convex sets. The word "maximal" in the theorem's statement refers to the set-inclusion order relation.

Theorem 1. Let $S$ be a compact subset of the locally convex space $X$, such that the interior of $S$ is dense in $S$. There exists a partition of intS formed by maximal convex subsets, having in common at most parts of the boundaries. If the space $S$ is separable, the partition is at most countable.

Proof. We give the proof only for the separable case. Let $\left\{x_{n}\right\}_{n \in \mathrm{N}} \subset S$ be a dense subset and assume $x^{0}$ to be a point of intS. Denote by $C\left(x^{0}\right)$ the family of all convex, closed subsets of $S$, containing $x^{0}$ in their relative interior with respect to $S$. Endow the nonempty family $C\left(x^{0}\right)$ with the order relation defined by set inclusion relation. Every totally ordered family $\left(K_{j}\right)_{j} \subset C\left(x^{0}\right)$ is bounded from above by

$$
K=\operatorname{cl}\left(\bigcup_{j \in J} K_{j}\right) \in C\left(x^{0}\right) .
$$

The application of Zorn lemma yields the existence of a maximal element $M\left(x^{0}\right)$ in $C\left(x^{0}\right)$. Choose $x^{k_{1}} \in S \backslash M\left(x^{0}\right)$, where $k_{1}$ is the smallest integer such that $x^{k_{1}} \notin M\left(x^{0}\right)$. There is a closed convex neighborhood $V\left(x^{k_{1}}\right)$ of $x^{k_{1}}$ in $S$, that is not intersecting $M\left(x^{0}\right)$. Application of Zorn lemma to the family of all closed convex subsets containing $x^{k_{1}}$ in their interior, such that the intersection of their interior with the interior of $M\left(x^{0}\right)$ is empty, leads to a maximal element $M\left(x^{1}\right) \in C\left(x^{1}\right)$, and so on. The step $n$ consists of application of Zorn lemma to the family of convex closed subsets not intersecting the subset

$$
\bigcup_{j \leq n-1} \operatorname{int}\left(M\left(x^{k_{j}}\right)\right)
$$

and which contain $x^{k_{n}}$, where $k^{n}$ is the smallest natural number such that

$$
x^{k_{n}} \notin \bigcup_{j \leq n-1} M\left(x^{k_{j}}\right) .
$$

Thanks to the density of the sequence $\left\{x^{n}\right\}_{n \in \mathrm{N}}$ in $S$, the above considerations lead to the existence of a subsequence $\left(x^{k_{n}}\right)_{n \in \mathrm{N}}$ such that

$$
\operatorname{cl}\left(\bigcup_{n \in \mathrm{N}} M\left(x^{k_{n}}\right)\right)=S, m \neq n \Rightarrow \operatorname{int}\left(M\left(x^{k_{m}}\right)\right) \cap \operatorname{int}\left(M\left(x^{k_{n}}\right)\right)=\Phi .
$$

Hence, $\left\{M\left(x^{k_{n}}\right)\right\}_{n}$ form a "convex partition" of the interior of $S$, with the properties mentioned in the statement. From a geometric point of view, there are "large" convex components having the barycenters situated far from the boundary. Some components may be "thin" convex components, having the sequence of barycenters converging to a boundary point. This concludes the proof. $\diamond$

\section{On Minimal Length and Minimal Area of Surrounding Curves and Surfaces}

Let $K \subset R^{2}$ be a simply connected compact subset defined by

$$
K=\{F \leq 1\}, \partial K=\{F=1\}, F \in C^{2}\left(R^{2}\right), \nabla F(x) \neq \mathbf{0} \quad \forall x \in K \backslash S,
$$


where $S$ is the set of minimum points of $F$ situated in $K$. Consider the problem of surrounding $K_{\varepsilon}=\{x ; d(x, K) \leq \varepsilon\}$ by a closed path $\gamma$ of minimal length. A variation of the problem is determining only "a half" of such a path. The problem has practical significance.

Theorem 2. Let $C=\operatorname{conv}\left(K_{\varepsilon}\right)=\operatorname{conv}\left(K_{\varepsilon}\right)$ be the (closed) convex hull of subset $K_{\varepsilon}$. Then the path $\gamma=\partial C$ is the shortest curve surrounding $K_{\varepsilon}$. The concave (respectively convex) "branches" defined by this path are uniformly approximated by concavity (convexity) preserving polynomials.

Proof. Due to Caratheodory’s theorem ([6], p. 10), or [7], we have:

$$
C=\left\{\sum_{j=1}^{3} \alpha_{j} x_{j} ; x_{j} \in \operatorname{Extr}\left(K_{\varepsilon}\right), \alpha_{j} \in R_{+}, \sum_{j=1}^{3} \alpha_{j}=1\right\}
$$

so that $C:=\operatorname{conv}\left(K_{\varepsilon}\right)$ is not only convex, but also compact. The geometric meaning of the above equality is that constructing $C$ is equivalent to joining some "convex components" to $K_{\varepsilon}$, wherever the latter subset is not convex. Recall that for a finite dimensional compact subset $K$ one has $\operatorname{co}(K)=\operatorname{co}(\operatorname{Extr}(K))$. This is a consequence of Caratheodory's theorem too. In order to form the boundary of the convex hull, we replace the arches of "non-convexity” by line segments. Any point

$$
x \in C \backslash K_{\varepsilon}
$$

lies in a triangle having such a line segment as one of the edges, with extreme points of $K_{\varepsilon}$ as the segment's ends. The third vertex of this triangle is also an extreme point in $K_{\varepsilon}$. Clearly, $\gamma=\partial C$ does not intersect the interior of $K_{\varepsilon} \subset C$. From the above arguments, the path $\gamma$ is formed by joining the arches of $\partial K_{\varepsilon}$ with the new added line segments as described above. Because these line segments represent the shortest path between the segment's ends, and because obviously $\gamma$ surrounds $C \supset K_{\varepsilon}$, the first assertion in the statement follows. The points at which the Hessian of $F$ is positive semidefinite remain unchanged when constructing the convex hull of $K_{\varepsilon}$.

Consider the concave function having as graph the "upper branch" and the convex function defined by the "lower branch" of $\gamma$. In order to avoid non-smooth paths, the function for the "upper branch" is approximated by the corresponding Bernstein polynomials, which preserve the concavity of any concave continuous function. By also approximating the convex function defined by the "lower branch" of $\gamma$ with Bernstein polynomials, the conclusion follows. $\diamond$

Remark 3. The following variant of Theorem 2 is natural and useful in applications, due to its iterative character: the boundary of $K_{\varepsilon}$ is usually a continuous piecewise smooth curve, which is uniformly approximated by polygonal lines. Given a non-convex polygon, it is easy to describe an algorithm of constructing its convex hull, simply dropping out some edges and vertices of "non-convexity". The convex hull of $K_{\varepsilon}$ is approximated by such convex polygons.

The method from theorem 2 works for compact subsets $K \subset R^{n}$, defined by means of a $C^{2}$-function on $R^{n}$, similarly to (1).

Theorem 4. Let consider a compact subset $K \subset R^{n}, n \geq 3, K_{\varepsilon}=\{x ; d(x, K) \leq \varepsilon\}$. Then $\partial\left(\operatorname{conv}\left(K_{\varepsilon}\right)\right)$ has a minimum surface area among hypersurfaces surrounding $K_{\varepsilon}$.

Proof. One uses Caratheodory's theorem. The $n-1$ dimensional simplexes, which compose part of $\partial\left(\operatorname{conv}\left(K_{\varepsilon}\right)\right)$ have smaller surface-area than any other hypersurfaces containing their vertices. The other part of $\partial\left(\operatorname{conv}\left(K_{\varepsilon}\right)\right)$, where the Hessian of $F$ is positive semidefinite, is part of $\partial K_{\varepsilon}$ as well. If the boundary of $\operatorname{conv}\left(K_{\varepsilon}\right)$ is not smooth, it can be approximated by smooth hypersurfaces [8]. $\diamond$

\section{Approximating the Perimeter of the Ellipse}

We start by proposing an exact formula for the perimeter of an ellipse

$$
x^{2} / a^{2}+y^{2} / b^{2}=1, a / \sqrt{5}<b<a,
$$

by means of an alternate series. The motivation is approximating the perimeter, with the control of the error.

Theorem 5. If $1 / \sqrt{5}<b / a$, the perimeter $l_{E}$ of the ellipse is

$$
l_{E}=2 b \pi\left[1+\frac{c^{2}}{4 b^{2}}+\left(\sum_{n=2}^{\infty} \frac{\psi^{(n)}(0)}{n !} \cdot\left(\begin{array}{c}
2 n \\
n
\end{array}\right) \cdot\left(\frac{c^{2}}{4 b^{2}}\right)^{n}\right)\right], c^{2}=a^{2}-b^{2}, \psi(z)=(1+z)^{1 / 2},|z|<1 .
$$


Proof. The following computations hold

$$
\begin{aligned}
l_{E}= & 4 \int_{0}^{\pi / 2}\left(a^{2} \sin ^{2} t+b^{2} \cos ^{2} t\right)^{1 / 2} \mathrm{~d} t=4 b \int_{0}^{\pi / 2}\left(1+\frac{c^{2}}{b^{2}}\left(1-\cos ^{2} t\right)\right)^{1 / 2} \mathrm{~d} t \\
= & 2 b \int_{R}\left(1+\frac{c^{2}}{b^{2}} \cdot \frac{u^{2}}{1+u^{2}}\right)^{1 / 2}\left(1+u^{2}\right)^{-1} \mathrm{~d} u \\
= & 2 b \int_{R}\left(\frac{1}{1+u^{2}}\right) \mathrm{d} u+2 b \cdot \frac{1}{2} \cdot \frac{c^{2}}{b^{2}} \cdot \int_{R} \frac{u^{2}}{\left(1+u^{2}\right)^{2}} \mathrm{~d} u+\cdots \\
& +2 b \cdot(-1)^{n-1} \cdot \frac{(2 n-3) ! !}{(2 n) ! !} \cdot \frac{c^{2 n}}{b^{2 n}} \cdot \int_{R} \frac{u^{2 n}}{\left(1+u^{2}\right)^{n+1}} \mathrm{~d} u+\cdots, \quad u=\operatorname{tg} t .
\end{aligned}
$$

Each of the integrals in the last sum is computed below using residues theorem on the contour formed by a line segment $[-R, R]$ of the real axes and the upper semicircle of radius $R$. Due to Jordan's lemma, the integral on the semicircle converges to zero as $R \rightarrow \infty$. Residues formula and Leibniz rule for derivation of a product yield

$$
\begin{aligned}
\int_{R} \frac{u^{2 n}}{\left(1+u^{2}\right)^{n+1}} \mathrm{~d} u & =2 \pi i \cdot \frac{1}{n !} \frac{\mathrm{d}^{n}}{\mathrm{~d} z^{n}}\left(\frac{z^{2 n}}{(z+i)^{n+1}}\right)_{\mid z=i}\left[1-\frac{\left(\begin{array}{c}
n \\
1
\end{array}\right)}{2}+\cdots+(-1)^{k} \cdot \frac{\left(\begin{array}{l}
n \\
k
\end{array}\right)}{2^{k}}+\cdots+\frac{(-1)^{n}}{2^{n}}\right] \\
& \left.=\frac{2 \pi i(2 n)(2 n-1) \cdots(n+1)}{2^{n+1} n ! \cdot i}\right] \\
& =\frac{\pi}{2^{2 n} n !}(2 n)(2 n-1) \cdots(n+1)=\frac{\pi}{4^{n}}\left(\begin{array}{c}
2 n \\
n
\end{array}\right) .
\end{aligned}
$$

Substitution of these values of the integrals into the series giving the perimeter of the ellipse leads to

$$
\begin{aligned}
l_{E} & =2 b \pi\left[1+\frac{c^{2}}{2 b^{2}} \cdot \frac{1}{2}+\cdots+(-1)^{n-1} \frac{(2 n-3) ! !}{(2 n) ! !} \cdot \frac{c^{2 n}}{4^{n} b^{2 n}} \cdot\left(\begin{array}{c}
2 n \\
n
\end{array}\right)+\cdots\right] \\
& =2 b \pi\left[1+\frac{c^{2}}{4 b^{2}}+\cdots+\frac{\psi^{(n)}(0)}{n !} \cdot\left(\begin{array}{c}
2 n \\
n
\end{array}\right) \cdot\left(\frac{c^{2}}{4 b^{2}}\right)^{n}+\cdots\right]
\end{aligned}
$$

Thus (2) is proved. This concludes the proof. $\diamond$

The next results concern the approximation of the perimeter of the ellipse by means of homogeneous inequalities.

Theorem 6. The following estimations hold

$$
4\left(a^{2}+b^{2}\right)^{1 / 2} \leq l_{E} \leq 2 \pi\left(a^{4}+b^{4}\right)^{1 / 4}
$$

Proof. One uses the following form of Jensen's inequality ([9], Section 2.7.2). For any convex positive homogeneous function $\varphi: R_{+} \times R_{+} \rightarrow R_{+}$, any positive Radon measure $\mu$ on a Hausdorff locally compact space $S$, and all $\mu$-integrable functions $f_{1}, f_{2}$ on $S$ one has:

$$
\int_{S} \varphi\left(f_{1}(t), f_{2}(t)\right) \mathrm{d} \mu(t) \geq \varphi\left(\int_{S} f_{1}(t) \mathrm{d} \mu(t), \int_{S} f_{2}(t) \mathrm{d} \mu(t)\right) .
$$

Applying this to

$$
S=[0, \pi / 2], \mathrm{d} \mu=\mathrm{d} t, \varphi(x, y)=\left(a^{2} x^{2}+b^{2} y^{2}\right)^{1 / 2}, f_{1}(t)=\sin t, f_{2}(t)=\cos t,
$$

one obtains: 


$$
l_{E}=4 \int_{[0, \pi / 2]}\left(a^{2} \sin ^{2} t+b^{2} \cos ^{2} t\right)^{1 / 2} \mathrm{~d} t \geq 4\left(a^{2}\left(\int_{[0, \pi / 2]} \sin t\right)^{2}+b^{2}\left(\int_{[0, \pi / 2]} \cos t\right)^{2}\right)^{1 / 2}=4\left(a^{2}+b^{2}\right)^{1 / 2}
$$

The second relation is a consequence of Schwarz inequality. $\diamond$

The next result gives evaluation formulae of the perimeter of the ellipse, involving "In"-function. We start by recalling the following well-known expansion-formula, based on the binomial series too. This time the series is not alternate. One has

$$
\begin{aligned}
& l_{E}=4 a \int_{0}^{\pi / 2}\left(1-\bar{e}^{2} \sin ^{2} t\right)^{1 / 2} \mathrm{~d} t=2 a \pi\left(1-\frac{\bar{e}^{2}}{4}-\cdots-\frac{((2 n-1) ! !)^{2}}{((2 n) ! !)^{2}} \cdot \frac{\bar{e}^{2 n}}{2 n-1}-\cdots\right), \\
& \bar{e}:=\left(\frac{a^{2}-b^{2}}{a^{2}}\right)^{1 / 2}<1,0<b<a .
\end{aligned}
$$

Theorem 7. The following evaluations hold

$$
2 a \pi-2 a \bar{e} \ln \left(\frac{1+\bar{e}}{1-\bar{e}}\right)-2 a \ln \left(1-\bar{e}^{2}\right) \leq l_{E} \leq 2 a \pi-2 a+a\left(\bar{e}^{-1}-\bar{e}\right) \ln \left(\frac{1+\bar{e}}{1-\bar{e}}\right)
$$

Proof. One uses the inequalities related to Wallis' relations:

$$
\frac{1}{2 n+1}<\left(\frac{(2 n-1) ! !}{(2 n) ! !}\right)^{2} \cdot \frac{\pi}{2}<\frac{1}{2 n}, n \geq 1 .
$$

Multiplying the first of the inequalities (4) with $-4 a \bar{e}^{2 n} /(2 n-1)$ and inserting into (3) yield:

$$
\begin{aligned}
l_{E} & <4 a\left[\frac{\pi}{2}-\sum_{n=1}^{\infty} \frac{\bar{e}^{2 n}}{(2 n-1)(2 n+1)}\right] \\
& =4 a\left[\frac{\pi}{2}-\frac{\bar{e}}{2}\left(\sum_{n=1}^{\infty} \frac{\bar{e}^{2 n-1}}{2 n-1}\right)+\frac{1}{2 \bar{e}}\left(\sum_{n=1}^{\infty} \frac{\bar{e}^{2 n+1}}{2 n+1}\right)\right] \\
& =4 a\left[\frac{\pi}{2}-\frac{\bar{e}}{2} \cdot \int_{0}^{\bar{e}}\left(1+t^{2}+\cdots\right) \mathrm{d} t+\frac{1}{2 \bar{e}} \int_{0}^{\bar{e}}\left(t^{2}+t^{4}+\cdots\right) \mathrm{d} t\right] \\
& =2 a \pi-2 a+a\left(\bar{e}^{-1}-\bar{e}\right) \ln \left(\frac{1+\bar{e}}{1-\bar{e}}\right) .
\end{aligned}
$$

The first relation of the statement one proves in a similar way. Namely, multiplying by $-4 a \bar{e}^{2 n} /(2 n-1)$ the second inequality (4), one obtains, also using (3)

$$
\begin{aligned}
-\frac{4 a \bar{e}^{2 n}}{2 n \cdot(2 n-1)}<-\pi \frac{2 a \bar{e}^{2 n}}{2 n-1} \cdot\left(\frac{(2 n-1) ! !}{(2 n) ! !}\right)^{2} \\
\Rightarrow l_{E} \geq 2 a \pi-4 a \cdot\left(\sum_{n=1}^{\infty} \frac{\bar{e}^{2 n}}{2 n \cdot(2 n-1)}\right) \\
\quad=2 a \pi-4 a \bar{e} \cdot\left(\sum_{n=1}^{\infty} \frac{\bar{e}^{2 n-1}}{2 n-1}\right)+4 a \cdot\left(\sum_{n=1}^{\infty} \frac{\bar{e}^{2 n}}{2 n}\right) \\
\quad=2 a \pi-4 a \bar{e} \cdot \int_{0}^{\bar{e}}\left(1+t^{2}+\cdots+t^{2 n-2}+\cdots\right) \mathrm{d} t+4 a \cdot \int_{0}^{\bar{e}}\left(t+t^{3}+\cdots+t^{2 n-1}+\cdots\right) \mathrm{d} t \\
\quad=2 a \pi-2 a \bar{e} \cdot \ln \left(\frac{1+\bar{e}}{1-\bar{e}}\right)-2 a \cdot \ln \left(1-\bar{e}^{2}\right) .
\end{aligned}
$$

This concludes the proof. $\diamond$ 
Theorem 8. Let $y_{G}$ be the ordinate of the gravity center of the upper semiellipse. Then for fixed $b>0$, we have:

$$
\lim _{a \rightarrow \infty} y_{G}(a, b)=b \frac{\pi}{4}, \lim _{a \rightarrow 0} y_{G}(a, b)=b / 2 .
$$

Proof. A straightforward application of the Guldin's formula leads to:

$$
A(S)=2 \pi b\left[b+\frac{a^{2}}{c} \sin ^{-1}(c / a)\right]=2 \pi \cdot y_{G} \cdot \frac{l_{E}}{2}=\pi \cdot l_{E} \cdot y_{G},
$$

where $A(S)$ is the area of the rotation surface obtained by rotation of the upper semiellipse around $O x$ axes, $c^{2}=a^{2}-b^{2}$. From this formula, we derive

$$
y_{G}=\frac{2 b\left[b / a+\frac{a}{c} \sin ^{-1}(c / a)\right]}{\int_{-\pi}^{\pi}\left(\sin ^{2} \theta+\left(b^{2} / a^{2}\right) \cos ^{2} \theta\right)^{1 / 2} \mathrm{~d} \theta} .
$$

When $a \rightarrow \infty, b / a \rightarrow 0, c / a \rightarrow 1$, so that an application of Lebesgue dominated convergence theorem yields:

$$
\lim _{a \rightarrow \infty} y_{G}(a, b)=\frac{2 b \cdot(\pi / 2)}{4}=\frac{b \pi}{4} .
$$

Next one considers the case $a \rightarrow 0$. The formula for $y_{G}$ is

$$
y_{G}=\frac{b \cdot \int_{-a}^{a}\left(1-\frac{x^{2}}{a^{2}}+\frac{b^{2}}{a^{2}} \cdot \frac{x^{2}}{a^{2}}\right)^{1 / 2} \mathrm{~d} x}{\int_{-a}^{a}\left(1+\frac{b^{2}}{a^{2}} \cdot\left(\frac{x^{2}}{a^{2}} /\left(1-\frac{x^{2}}{a^{2}}\right)\right)\right)^{1 / 2} \mathrm{~d} x}=\frac{b \int_{0}^{\pi / 2}\left(1-\cos ^{2} \theta+\frac{b^{2}}{a^{2}} \cos ^{2} \theta\right)^{1 / 2} \sin \theta \mathrm{d} \theta}{\int_{0}^{\pi / 2}\left(\sin ^{2} \theta+\frac{b^{2}}{a^{2}} \cos ^{2} \theta\right)^{1 / 2} \mathrm{~d} \theta} .
$$

Next, we multiply the nominator and the denominator by the scale ratio $a / b \rightarrow 0$. Application of the Lebesgue dominated convergence theorem yields

$$
\lim _{a \rightarrow 0} y_{G}(a, b)=\frac{b \cdot \int_{0}^{\pi / 2} \cos \theta \cdot \sin \theta \mathrm{d} \theta}{\int_{0}^{\pi / 2} \cos \theta \mathrm{d} \theta}=b / 2 .
$$

This concludes the proof. $\diamond$

\section{Conclusion}

We construct a convex partition of an arbitrary compact subset, similarly to decomposition of an open subset into its connected components. Minimal-length surrounding curves and minimal-area surrounding surfaces are constructed too. Next, one deduces a formula for the perimeter of the ellipse, as the sum of an alternate series. Consequently, one can evaluate the error easily in approximating by partial sums. One proves two other approximation results based on appropriate "sandwich" type inequalities. We discuss the ordinate of the upper semiellipse at the ends of the positive semiaxes in terms of the ratio $b / a, b$ fixed, $a \in(0, \infty)$.

\section{References}

[1] Almkvist, G. and Berndt, B. (1988) Gauss, Landen, Ramanujan, the Arithmetic Geometric Mean, Ellipses, $\pi$, and the Ladies Diary. The American Mathematical Monthly, 95, 585-608. http://dx.doi.org/10.2307/2323302

[2] Chandrupatla, T.R. and Osler, T.J. (2010) The Perimeter of an Ellipse. The Mathematical Scientist, 35, $122-131$.

[3] Barnard, R.W., Pearce, K. and Schovanec, L. (2001) Inequalities for the Perimeter of an Ellipse. Journal of Mathematical Analysis and Applications, 260, 295-306. http://dx.doi.org/10.1006/jmaa.2000.7128

[4] Neumann, M. (1977) On the Strassen Disintegration Theorem. Archiv der Mathematik, 29, 413-420. http://dx.doi.org/10.1007/BF01220429 
[5] Udrişte, C., Ţevy, I. and Arsinte, V. (2010) Minimal Surfaces between Two Points. Journal of Advanced Mathematical Studies, 3, 105-116.

[6] Phelps, R.R. (1966) Lectures on Choquet's Theorem. D. Van Nostrand Company, Inc., Princeton.

[7] Boboc, N. and Bucur, Gh. (1976) Convex Cones of Continuous Functions on Compact Spaces. Academiei, Bucharest (in Romanian).

[8] Deville, R., Fonf, D. and Hájek, P. (1998) Analytic and Polyhedral Approximation of Convex Bodies in Separable Polyhedral Banach Spaces. Israel Journal of Mathematics, 105, 139-154. http://dx.doi.org/10.1007/BF02780326

[9] Niculescu, C. and Popa, N. (1981) Elements of Theory of Banach Spaces. Academiei, Bucharest (in Romanian).

[10] Olteanu, O. (1994) Uniform Approximation of Certain Continuous Functions. Studii si Cercetari Matematice (Mathematical Reports), 46, 533-541.

[11] Rudin, W. (1987) Real and Complex Analysis. 3rd Edition, McGraw-Hill, Inc., New York. 